\title{
Effect of Gradual Heating of Gold Nanoparticle Multilayers on Polymer Substrates on the Characteristics of their LSPR Bands
}

\author{
Michael Fanous, Simona Badilescu, Muthukumaran Packirisamy \\ Concordia University \\ Montreal, Canada \\ Optical-Bio Microsystems Laboratory, Mechanical and Industrial Engineering Department \\ 1515 Ste-Catherine Street West, Montréal, Qc, Canada H3G 2W1
}

\begin{abstract}
Polymer materials have become increasingly useful for photonic sensing applications. Their consistency and optical properties make them highly desirable for sensing possibilities. Bio-molecules, such as proteins, can be attached to a polymer substrate using a thin layer of gold nanoparticle. Gold nanoparticles (GNP) hold unique localized plasmon resonance (LSPR) properties, which are affected by their structure, shape, distribution, and their degree of penetration into the surrounding medium. As well, the dielectric properties of the medium influence LSPR responses. These characteristics, in turn, depend on the thermal history of the sample, that is, the extent and duration of heating of the polymer-GNP systems. In this work, we examine the specific effects of gradually increasing temperature through incremental heating on the LSPR response of GNP on various polymers, including cyclic olefin copolymer (COC), poly (methyl methacrylate) (PMMA), poly (dimethyl siloxane) (PDMS), SU-8, polycarbonate (PC) and polystyrene (PS). GNP on polymers manifested a shift in the LSPR peak response from $7 \mathrm{~nm}$ to $34 \mathrm{~nm}$ on average, and an important change in absorbance as well. These results will help enhance the sensing performance of microphotonic sensors by improving the shape of the LSPR band and selecting the ideal polymer material for specific applications. The information will also benefit other applications, including cell culture and biomolecular separation in a microfluidic environment. Furthermore, the findings herein may be employed towards developing a unidirectional temperature sensor.
\end{abstract}

Keywords: Gold nanoparticles; polymer; microfluidics; microphotonics; sensing

\section{Introduction}

During the last decennia, gold nanoparticles have widely been employed for sensing applications. The use of temperature to improve such endeavors has also been explored. It was found that with optical illumination, gold nanoparticles engender heat [1]. This lead to several exploitations, one of which was imaging proteins with gold nanoparticles in cells. The heating of gold nanoparticles has therefore been considered a possible source of improved sensitivity of the platforms [2].

The properties of a material's surface differ from those of its bulk volume as a result of split or tense bonds. The effect is especially marked in polymers, as the discrepancies at the surface are considerable. One of the affected mechanical properties is the glass transition temperature. Multiple examinations on polymer surfaces by various groups discovered that the surface transition temperature differs from the bulk transition temperature [3]. In 2003, Teichroeb and Forrest obtained images of embedded gold nanoparticles in a polystyrene surface using atomic force microscopy (AFM) [4]. The result indicated that there is a more malleable region of material at the surface, about 3-4 $\mathrm{nm}$ in thickness. This leads to the inference that there is a lower transition temperature at the surface compared to the bulk region. One expected outcome of heating is the penetration or sinking of the nanoparticles into the polymer, foreseen to generate a spectral shift in LSPR. This is in part a consequence of the alteration of the dielectric environment neighboring the nanoparticles, which become more enveloped in the underlying polymer layer the further they sink [5].

Surface Plasmon absorption bands are influenced by a number of factors of the material involved, such as its dimensions, shape, distribution, and surrounding environment. These facets may be manipulated in favor of improving the sensing capabilities by spectroscopy [5]. One use of this involves the adsorption of double-stranded DNA onto gold nanoislands, with the shift of the LSPR band being pronounced by binding gold nanoparticles of $12 \mathrm{~nm}$ diameter, onto the complementary single-stranded DNA [6].

The plasmon resonance may also be manipulated through the polarity of the materials concerned $[7,8]$. Another possible facet to be manipulated is the degree of aggregation of the particles. It was also found that ameliorations on the limit 
of detection through further defining of the resonance peak might be accomplished by using nanorods rather than nanospheres [9].

The way that heating in advance is implemented involves the transition temperatures of the materials. Since it was shown that the polymer surface layer has a glass transition temperature that is less than the commensurate bulk value, during the heating of polymer layers with a deposit of gold nanoparticles within the temperature range of $\mathrm{Tg}, \mathrm{s}<\mathrm{T}<\mathrm{Tg}$, the nanoparticles sink into in the surface layer of the polymer by an extent proportional to duration and intensity of heating [10].

Resonance of gold nanospheres takes place in the visible spectral region, at roughly $520 \mathrm{~nm}$, which is the source of the crimson hue of the nanoparticles in solution [11, 12]. The maximum of the surface plasmon band is also affected by the alterations to the polymer-nanoparticle arrangement that transpire during the course of heat treatment. Additionally, the refractive index of the surrounding medium has been found to provoke a shift of the surface plasmon band, described by Mie theory [13].

The central objective of this work is to ameliorate the sensing performance of microphotonic sensors. The results obtained herein will contribute to improving the shape of the LSPR band, polymer-protein affinity, and the optimization of the polymer material for specific applications. Such findings will also assist in various other applications, including cell culture, bio-molecular separation, and the identification and study of biological molecules, which is of paramount medical importance [14]. For instance, gold-polymer nanocomposites have been found to be fruitful in the detection of biomedical entities, such as in the detection of growth hormones on a microfluidic chip [15].

\section{Materials and Methods}

The polymers investigated in this work are poly (methyl methacrylate) (PMMA), polystyrene (PS), SU-82, poly (vinyl alcohol) (PVA), poly (dimethyl siloxane) (PDMS), and cyclic olefin copolymer (COC). The polymers were first spin-coated on small glass slides and thereafter annealed for a time that is suitable to their consistencies. For example, PDMS was heated incrementally for a total of two hours at $100{ }^{\circ} \mathrm{C}$ and PS was heated for five hours at $60{ }^{\circ} \mathrm{C}$.

Gold nanoparticles were deposited from a colloidal solution on the polymer films through a thermal convection method, as shown in Figure 1. This technique entails the transfer of gold nanoparticles from the colloidal solution onto the polymer substrate through evaporation and assembly [16]. The gold nanoparticles were synthesized from $1 \mathrm{mM}$ of $\mathrm{HAuCl}_{4}$ by $\mathrm{using}$ a sodium citrate solution $1 \%$ as a reducing agent.

Heating of the polymer-Au system was done using the digitzed ThermoLyne oven, for periods of 10 minutes, in gradations of $25^{\circ} \mathrm{C}$.

PekinElmer's LAMBDA 650, a UV-visible spectrometer, was used to measure the absorbance of the samples, from wavelengths of $400 \mathrm{~nm}$ to $800 \mathrm{~nm}$, at various stages of previous heating. A scanning electron microscope was used to study the morphology and distribution of gold nanoparticles adsorbed onto the polymer films.

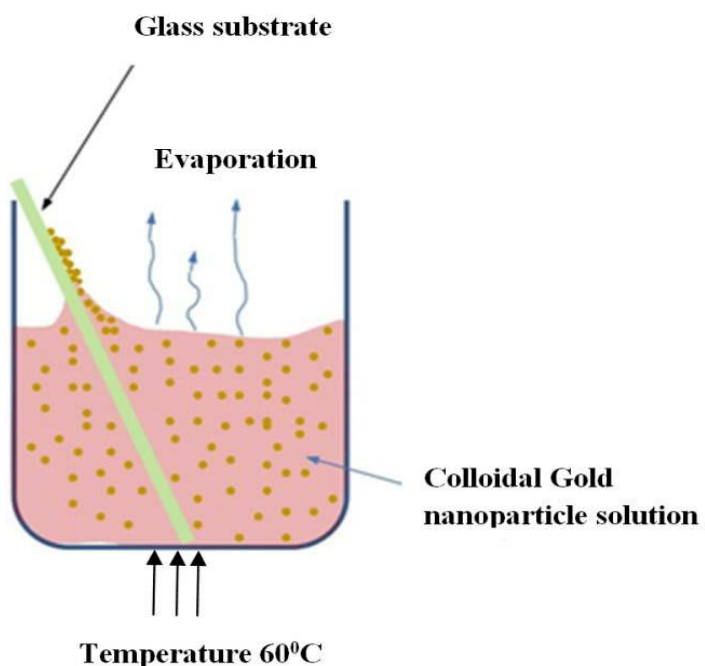

Fig.1: Schematic of the convective assembly procedure of deposition of gold nanoparticles [Reproduced with permission from 15].

\section{Results}




\subsection{Simulation}

A Finite-Difference Time-Domain (FDTD) simulation of the particles was created to analyze the effects of distribution and depth of particle alterations throughout the heating process. This property was assessed by emulating the localized surface plasmon resonance (LSPR) procedure, as shown in figure 2 (A) [17].

RSoft FullWAVE, a commercial software program for optical applications, was used for the design and testing of the polymer structures, which were approximated as spheres, and defined through the material bank of the program as Au.

To create the virtual reality of a sphere embedded in polymer, a merge priority was given to the particles over the films. The technical details of the simulation parameters are as follows: the perfectly matched layer (the boundary condition) is set to $100 \mathrm{~nm}$ in every direction, and is placed on the confines of the simulation domain, making the light to be entirely absorbed on the boundary without reflections. In this digital process, the gold nanosphere was excited by a source with plane waves emanating from a rectangular launch pad, having a transverse electric field. Additionally, a pulse excitation was selected to impinge the gold nanoparticles.
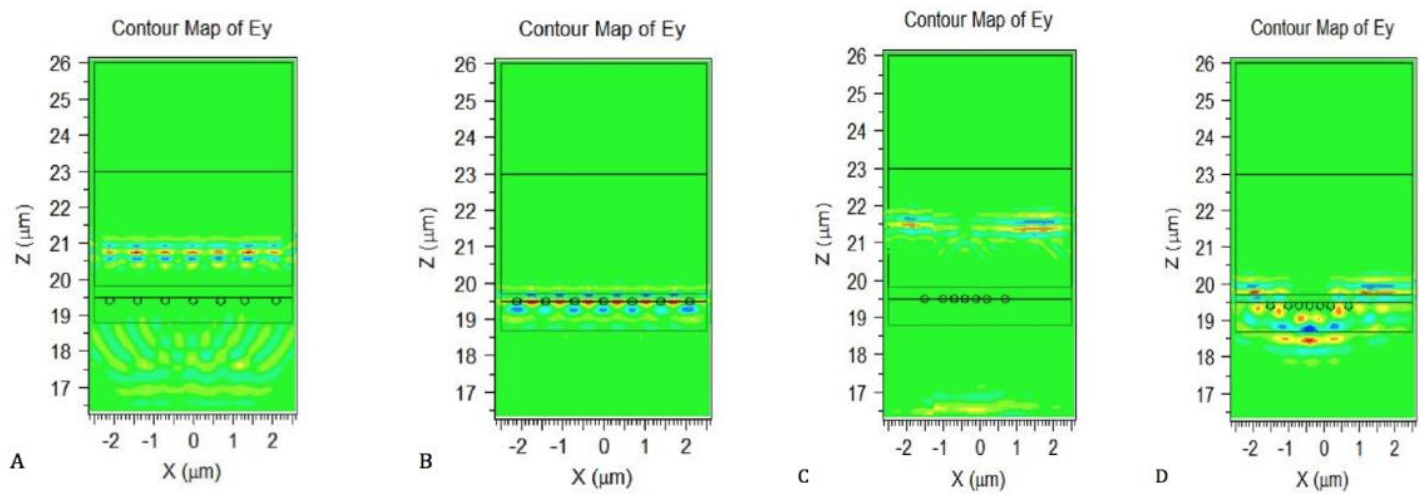

Fig. 2: FDTD simulations of the LSPR measurements of gold nanoparticles on a polymer: (A) evenly distributed particles not embedded; (B) evenly distributed particles that are embedded; (C) unevenly distributed particles embedded; (D) unevenly distributed particles that are not embedded.

These were contained in a monitor measuring absorbance from every direction. The electric field distribution on the configuration on the y direction, that is the cross section plane, was simulated by FDTD, and is shown in Figure 2. The absorbance spectrum of the gold nanoparticles was calculated through a Fast Fourier Transform (FFT) algorithm. The domain was divided into minute fragments, known as the 'grid'. The diminutiveness of this value determines the accuracy of the model. In this case, a value of $10 \mathrm{~nm}$ was selected

Four different configurations were tested to examine the gamut of possibilities. The first is a geometry involving nanoparticles, not to scale or relative proportion, over a polymer film, which itself sits on a glass slide. The particles are just peripherally in contact with the polymer. That is, they are not embedded. Additionally, they are perfectly distributed across the polymer surface. The three subsequent iterations follow from this arrangement, varying the dispersion of the particles, and the degree of their sinking. The simulation is launched and absorbance bands are generated. These are juxtaposed to contrast any discrepancies in shift. A slight shift rightward is observed for both the effect of unevenness and impregnation. In comparing the first band directly with each other band, it becomes apparent that either a change in distribution or particle depth causes an increase in wavelength of the peak absorbance value. Furthermore, the absorbance peak of 0.22 increases to 0.3 with uneven distribution of particles that are not embedded. The opposite effect, a slight dip in absorbance, occurs when the particles are embedded with even distribution, with a maximum in absorbance of 0.2.

\subsection{Morphology of GNPs on the surface of polymers}

The purpose of imaging the GNP-polymer systems is to discern the morphology and inter-distance changes among the particles on the surface of the polymer. Figure 3 shows the images of two of the investigated polymers (PVA and PDMS). The first image, Figure 3 (A), shows a film of PVA with a gold deposit after being gradually heated to $250{ }^{\circ} \mathrm{C}$. Metal particles appear as clustered forming filaments, indicating the linear aggregation of particles. The next image, Figure 3 (B), is that of PDMS bearing the gold coating, also heated up to $250{ }^{\circ} \mathrm{C}$. The density of GNP on PDMS appears higher and the image 
indicates a branching aggregation. The last two images, Figure 3 (C) and (D), show GNP on a glass slide, without any polymer and a PDMS film that did not undergo any heating, respectively. Here the particles appear much more sparse and spread out, in addition to being less strongly attached.
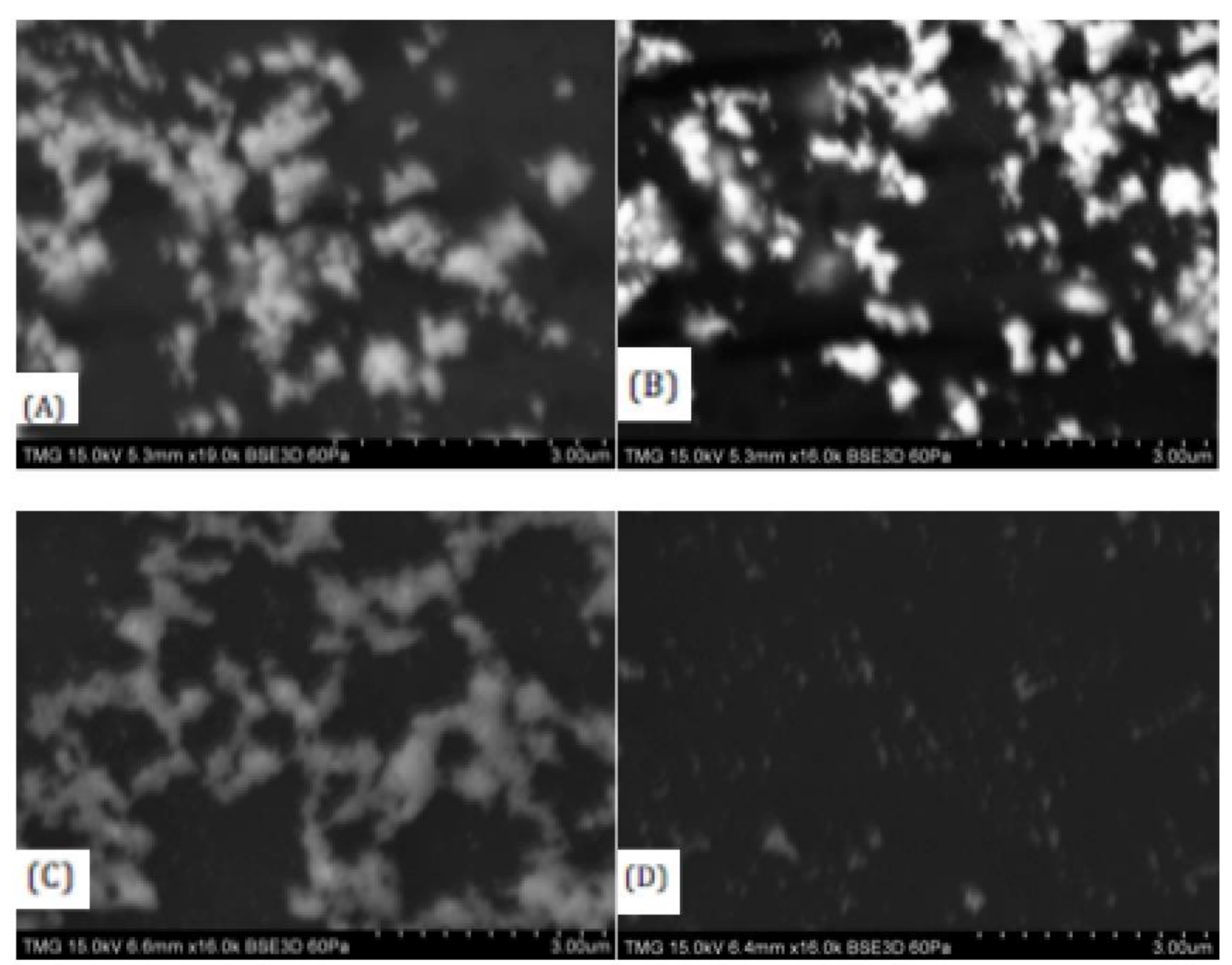

Fig. 3: SEM images of gold nanoparticles: (a) PVA after heating; (b) PDMS after heating; (c) glass slide without polymer and not subjected to heating; (d) PDMS not subjected to heating.

\subsection{Effect of gradual heating on the Au LSPR band}

The first series of bands, shown in Figure 4, correspond to a series of LSPR bands of the six polymers with gold, through the escalating stages of the heating process. These bands correspond to the Au nanoparticles on the respective polymers, along with the final shifts. These show a general shift of peak wavelength to longer wavelengths. It appears the LSPR bands corresponding to GNP on COC, SU-8, and PMMA polymers all underwent a peak shift of $34 \mathrm{~nm}$ corresponding to the interval between of heat treatment of $80{ }^{\circ} \mathrm{C}$ to $250{ }^{\circ} \mathrm{C}$. COC increased from roughly $575 \mathrm{~nm}$, SU-8 from $525 \mathrm{~nm}$, and PMMA from $520 \mathrm{~nm}$. The transition was mostly smooth, however the absorbance values dip decrease and then ascend, with those PMMA appearing particular far apart, with gaps of absorbance between measurement as large as 0.1. The PS film experienced a comparably slight shift of $7 \mathrm{~nm}$, with an increase from a starting value of $580 \mathrm{~nm}$, as well as downwards to upwards changes in absorbance. The major part of this transition appears to take place at the $200{ }^{\circ} \mathrm{C}$ mark. PDMS, in contrast, shows a shift of $12 \mathrm{~nm}$, increasing from $530 \mathrm{~nm}$, with the greatest difference taking place at $175^{\circ} \mathrm{C}$. These threshold temperatures may indicate the point at which the consistency of the polymer is sufficiently malleable to enable the greatest change in GNP configuration. Additionally, if one compares the thermo gravimetric curves of the polymers that experienced the greatest shifts, that is, PMMA, PVA, and SU-82, it will be apparent that they all share a mass change temperature of 250 $-300{ }^{\circ} \mathrm{C}$. Conversely, the polymers that experienced the slightest shifts, that is, COC, PS and PDMS, all have mass changes in a markedly higher range of $450-500{ }^{\circ} \mathrm{C}$. 

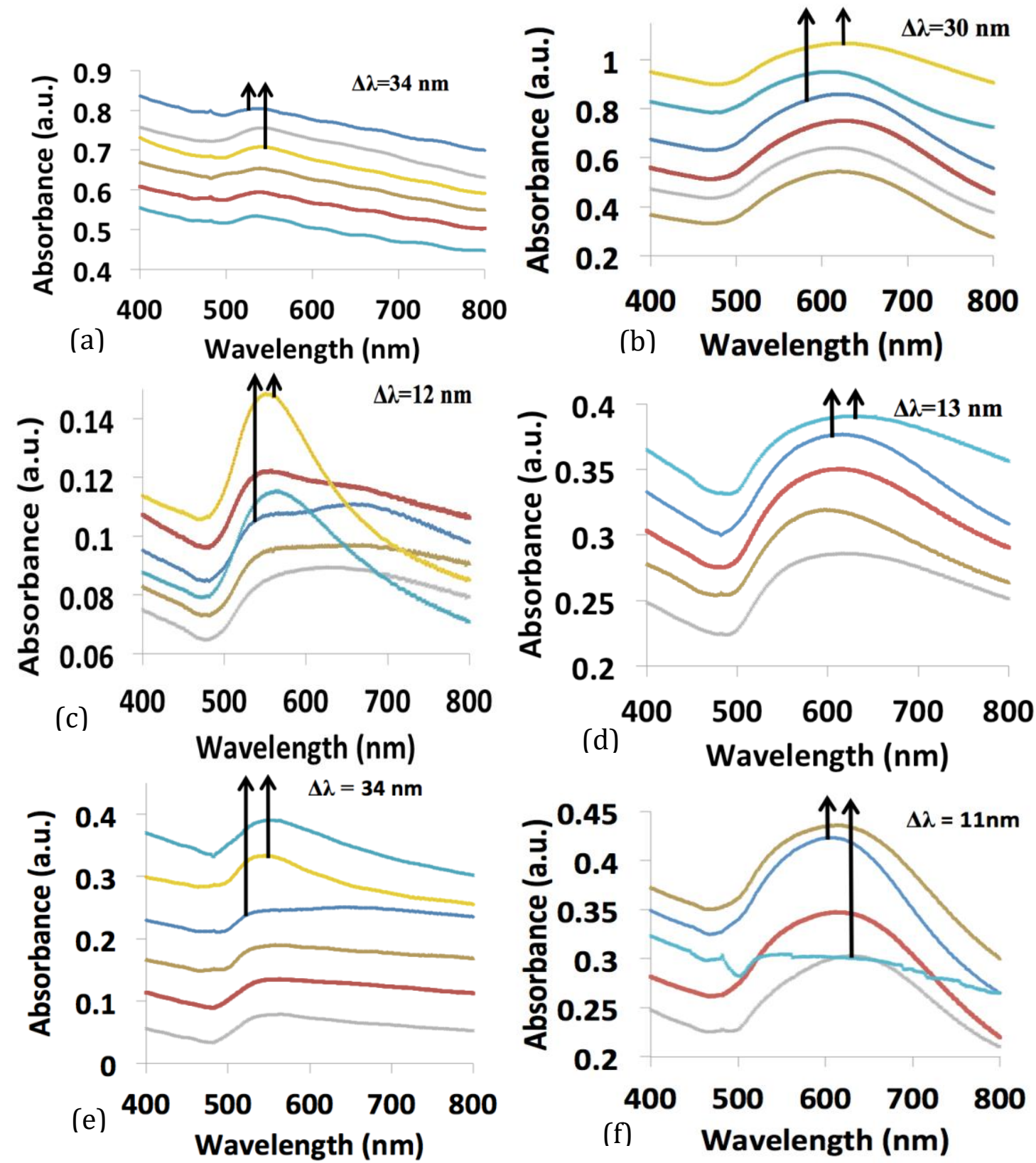

Fig. 4: Shift of the Au LSPR absorption bands corresponding to different polymers with temperature $\left(80{ }^{\circ} \mathrm{C} /\right.$ dark blue, $100{ }^{\circ} \mathrm{C} /$ red, $125{ }^{\circ} \mathrm{C} /$ brown, $150{ }^{\circ} \mathrm{C} /$ grey, $175^{\circ} \mathrm{C} /$ light blue, $200^{\circ} \mathrm{C} /$ yellow) (a) SU-82; (b) PVA; (c) PDMS; (d) PS; (e) PMMA; (f) COC.

The results show that the position of the Au LSPR bands of heated samples are at a higher wavelength than that of particles that are sitting on the surface of the polymer, as the bands move rightwards with increasing temperature overall. The band corresponding to the uneven distributions is more shifted than that of the even distributions. The comparison of the LSPR bands shows redshifts in wavelength peaks, and a change in the absorbance as well. The refractive indices generally decrease with temperature, and the wavelength peaks increase and then decrease with refractive indices, with a uniform peak at approximately 1.55. All the results agree with the morphology of the GNP aggregates as seen in the SEM images, with larger aggregates corresponding to heated samples. These distinct results may be attributed to decreasing refractive indices, embedding of particles, and greater polymer surface ratios [18]. 
Another method used to detect the difference in heated and non-heated samples was atomic force microscopy (AFM). As AFM employs a cantiliver tip to scan the sample vertically, it served to illustrate the degree of particle penetration into the polymer surface. The results show that, after the PVA sample had been heated gradually up to $225^{\circ} \mathrm{C}$, due sinking GNP, the roughness of the surface decreased.

\section{Conclusion}

The polymers showed the Au LSPR peak response (the shift) increase from $7 \mathrm{~nm}$ to $34 \mathrm{~nm}$ on average at a temperature range of $175-200^{\circ} \mathrm{C}$, and a general absorbance trend of downwards to upwards. The trend may be attributed to an initial aggregation (after deposition) and spreading of gold nanoparticles, and then, as they are increasingly embedded into the polymer surface, absorbance rises. The results are consistent and point towards the general effect of LSPR shifts toward longer wavelengths through aggregation and partial embedding by a gradual heating treatment. This is attributed to changing properties of both particle and polymer. The sinking particles incrementally change depth and degree of polymer envelopment during the course of heating. These changes effect a shift in the absorbance curves. The SEM results show heated samples with particles strongly attached to the polymer and aggregated, and the AFM images and graphs reveal a greater surface roughness for a non-heated sample, indicating a descent of particles into the polymer with heating.

The benefits of these observations, specific to each polymer, are the ability to refine sensing and affinity methods, while selecting the appropriate polymer material for a specific bio-sensing application, by implementing, in accordance with the analyte in question, a treatment of gradual heating in advance. Sensing experiments by using different polymer platforms as a substrate for gold nanoparticles are under way.

\section{Acknowledgments}

We are grateful to Dr. Jayan Ozhikandathil, Jalal Abdul-Hadi, and Dr. Pierre Pottier for their technical assistance in the laboratory set-ups and the RSoft simulation work.

\section{References}

[1] L. Cognet, C. Tardin, D. Boyer, D. Choquet, P. Tamarat, and B. Lounis, "Single metallic nanoparticle imaging for protein detection in cells," PNAS, vol. 100, pp. 11350-11355, 2003.

[2] S. Tokareva, J. Minko, H. Fendler, and E. Hutte, "Nanosensors based on responsive polymer brushes and gold nanoparticle enhanced transmission surface plasmon resonance spectroscopy," J. AM. CHEM. SOC., vol. 126, pp. 15950-15951, 2004.

[3] R. K. Putla, "Monitoring of glass transition at a polymer surface by localized surface plasmon resonance (thesis Oklahoma State University)," pp. 1-60, 2010.

[4] J. H. Teichroeb and J. A. Forrest, "Direct imaging of nanoparticle embedding to probe viscoelasticity of polymer surfaces," Phys. Rev. Lett., vol. 91, pp. 016104, 2003.

[5] M. Lahav, A. Vaskevich, and I. Rubinstein, "Biological sensing using transmission surface plasmon resonance spectroscopy," Am. Chem. Soc., vol. 20, pp. 7365-7367, 2004.

[6] E. Hutter and M. P. Pileni, "Hybridization of oligonucleotide-modified silver and gold nanoparticles in aqueous dispersions and on gold films," J. Phys, vol. 107, pp. 6497-6499, 2003.

[7] U. Kreibig and M. Vollmer, Optical Properties of Metal Clusters. Berlin, Germany: Springer, 1995.

[8] P. Mulvaney, "Surface plasmon spectroscopy of nanosized metal particles," Langmuir, vol. 12, pp. 788-800, 1996.

[9] C. Sönnichsen, T. Franzl, T. Wilk, Q. von Plessen, J. Feldmann, O. Wilson, and P. Mulvaney, "Drastic reduction of plasmon damping in gold nanorods," Phys. Rev. Lett., vol. 88, pp. 077402, 2002.

[10] Z. Adamczyk, P. Weronski, and E. Musial, "Metal nanoparticles on polymer surfaces: 3. Adsorption kinetics of gold hydrosol particles on polystyrene and poly(2-vinulpyridine)," J. Colloid Interface Sci., vol. 241, pp. 63, 2001.

[11] J. Yguerabide and E. E. Yguerabide, "Resonance light scattering particles as ultrasensitive labels for detection of analytes in a wide range of applications," J. Cell. Biochem. Suppl., vol. 37, pp. 71, 2001.

[12] G. Raschke, S. Kowarik, T. Franzl, C. Sonnichsen, T. A. Klar, and J. Feldmann, "Biomelucular recognition based on single gold nanoparticle light scattering," Nano letter., vol. 3, pp. 935-938, 2003.

[13]M.-C. Daniel and D. Astruc, "Gold nanoparticles: assembly, supermolecular chemistry, quantum-size-related properties, and applications toward biology, catalysis, and nanotechnology," Chem. Rev., vol. 104, pp. 293-346, 2004. 
[14] P. K. Jain, I. El-Sayed, and M. El-Sayed, "Gold nanoparticles: interesting optical properties and recent applications in cancer diagnostics and therapy," Future Medicine, vol. 2, pp. 681-693, 2007.

[15] J. Ozhikandathil, S. Badilescu, and M. Packirisamy, "Gold Nanoisland Structures Integrated in a Lab-on-a-Chip for Plasmonic Detection of Bovine Growth Hormone," J. Biomed. Opt., vol. 17, 2012.

[16] M. Fanous, J. Ozhikandathil, S. Badilescu, and P. Packirisamy, "Evaluation of optical properties and biocompatibility for polymer materials for microfluidic applications," Photonics North., pp. 35832, 2015.

[17] J. Ozhikandathil and M. Packirisamy, "Simulation and implementation of a morphology-tuned gold nano-islands integrated plasmonic sensor," Sensors, vol. 14, pp. 10497-10513, 2014.

[18] F. Amin, D. Yushchenko, F. Zhang, Z. Ali, and W.J. Parak, "Polarity sensitive colloidal fold nanoparticles," NSTINanotech., vol. 1, pp. 485- 487, 2010. 